\title{
Tabagismo e consumo de álcool em adolescentes de uma cidade de pequeno porte do Brasil
}

\author{
Smoking and alcohol consumption in adolescents from a small city in Brazil
}

Fumar y consumo de alcohol en adolescentes de una pequeña ciudad de Brasil

\begin{abstract}
Adelson Fernandes da Silva1*, Lais Castilho Xavier², Larissa Ferreira dos Santos², Maria Clara Álvaro Santos ${ }^{2}$, Salete Aparecida Duarte Figueiredo², Deyvid Rafael Dias Carvalho², Felipe Galdino Souza ${ }^{2}$, Nívea Maria de Oliveira Jacques ${ }^{1}$, Tiago Rodrigues de Lima ${ }^{3}$, Diego Augusto Santos Silva ${ }^{3}$.
\end{abstract}

\section{RESUMO}

Objetivo: Estimar a prevalência e os fatores sociodemográficos associados ao tabagismo e ao consumo de álcool em adolescentes escolares residentes em uma cidade de pequeno porte do Brasil. Métodos: Estudo transversal, conduzido em 583 escolares de 15 e 18 anos, de escolas públicas estaduais. Os dados foram coletados através do questionário "Estilo de Vida dos Adolescentes Manauara". Resultados: Maiores chances (OR: 4,26; IC95\%: 1,31-13,82) de ser ex-fumante foram verificadas nos escolares que residiam em áreas rurais. Além disso, ser do sexo masculino (OR: 5,18; IC95\%: 1,39-19,28), estar na faixa etária de 1718 anos (OR: 1,96; IC95\%: 1,16-3,30) e estudar no turno de estudo vespertino (OR: 7,66; IC95\%: 1,62$36,24)$ foi relacionado com maiores chances de ser fumante. Os adolescentes de faixa etária 17 à 18 anos tiveram 113\% mais chances de consumirem álcool de maneira excessiva (OR: 0,37; IC95\%: 0,25-2,78). Conclusão: A prevalência estimada para tabagismo foi inferior comparado ao consumo de álcool. Os subgrupos de adolescentes que tiveram maiores chances de fumar e consumir álcool, foram os escolares do sexo masculino com a faixa etária 18-19 anos.

Palavras-Chave: Álcool, Tabagismo, Escolares.

\begin{abstract}
Objective: To estimate the prevalence and sociodemographic factors associated with smoking and alcohol consumption in school adolescents living in a small Brazilian city. Methods: Cross-sectional study conducted in 583 schoolchildren aged 15 to 18 years of state public schools. Data were collected through the questionnaire "Estilo de Vida dos Adolescentes Manauara" (Lifestyle of Adolescents Manauara 2011). Results: Higher odds (OR: 4.26; 95\%Cl: 1.31-13.82) of being an ex-smoker were found in schoolchildren who lived in rural areas. In addition, being male (OR: 5.18; 95\% $\mathrm{Cl}: 1.39-19.28)$, being in the 17-18 age group (OR: 1.96; 95\%Cl: 1.16-3.30) and studying in the afternoon study shift (OR: 7.66; 95\%Cl: 1.62-36.24) was associated with greater odds of being a smoker. Adolescents aged 17 to 18 years were $113 \%$ more likely to consume alcohol excessively (OR: $0.37 ; 95 \% \mathrm{Cl}: 0.25-2.78$ ). Conclusion: The estimated prevalence for smoking was lower compared to alcohol consumption. The subgroups of adolescents who were more likely to smoke and consume alcohol, were male students aged 18-19 years.
\end{abstract}

Keywords: Alcohol, Smoking, Schooling.

\footnotetext{
1 Universidade Estadual de Montes Claros (UNIMONTES), Campus Januária - MG.

*E-mail: Adelson_fsilva@hotmail.com

2 Projeto Estágio não Obrigatório do Governo de Minas Gerais (UNIMONTES), Campus Januária - MG.

3 Universidade Federal de Santa Catarina (UFSC), Florianópolis - SC.
} 
RESUMEN

Objetivo: Identificar la prevalencia y factores asociados al consumo de alcohol y tabaco por escolares del 9응 año de la Escuela Primaria y del $1^{\circ}$ al $3^{\circ}$ año de la Escuela Secundaria de escuelas estaduales de la ciudad de Januária, Minas Gerais. Métodos: Este estudio fue de delineamiento transversal, la muestra fue construída por 583 escolares, con edades comprendidas entre 15 y 18 años. Para la recolección de datos, se utilizó el cuestionario "Estilo de Vida de los Adolescentes Manauara". Los datos fueron verificados por la prueba de Kolmogorov-Smirnov, donde los mismos presentaron una distribución no paramétrica $(p<0,05)$ y qui-quadrado ( $p=0,05)$. Resultados: Se encontraron mayores posibilidades (OR: 4.26; IC95\%: 1.31-13.82) de ser un exfumador en escolares que vivían en áreas rurales. Además, ser hombre (OR: 5.18; IC95\%: 1.39-19.28), estar en el grupo de edad 17-18 (OR: 1.96; IC95\%: 1.16-3.30) y estudiar en el turno de estudio de la tarde (OR: 7.66; IC95\%: 1.62-36.24) se relacionó con mayores posibilidades de ser fumador. Los adolescentes de 17 a 18 años tenían un $113 \%$ más de probabilidades de consumir alcohol en exceso (OR: 0,37; IC95\%: 0.25-2.78). Conclusión: La prevalencia estimada para fumar fue menor en comparación con el consumo de alcohol. Los subgrupos de adolescentes que tenían más probabilidades de fumar y consumir alcohol eran estudiantes varones de entre 18 y 19 años.

Palabras Clave: Alcohol, Tabaco, Escolares.

\section{INTRODUÇÃO}

O álcool e o tabaco são substâncias psicoativas, que se tornaram um importante problema de saúde pública no Brasil e no mundo. Na linguagem do dia-a-dia, dá-se o nome de álcool ao composto químico etanol, também chamado álcool etílico. Trata-se de um líquido incolor e inflamável cujo ponto de ebulição é de $78^{\circ} \mathrm{C}$ e tabaco é uma planta cujo nome científico é nicotiana tabacum, da qual extrai-se o princípio ativo, nicotina. É consumido como uma droga recreativa sob a forma de cigarro, charuto, cachimbo, rapé, narguilé, charro ou fumo mascado (CEBRID-UNIFESP, 2018). Essas substâncias atuam no sistema nervoso central, modificando o estado emocional e comportamental dos indivíduos, e é largamente utilizado por produzir sensações prazerosas (CARDOSO FM, et al., 2015).

O tabaco mata mais de 8 milhões de pessoas a cada ano. Mais de 7 milhões dessas mortes são resultado do uso direto do tabaco, enquanto cerca de 1,2 milhão são resultado de não-fumantes expostos ao fumo passivo (OPAS/OMS-BRASIL, 2019), O consumo de álcool, também é avassalador, pois é responsável por cerca de 3,3 milhões de mortes no mundo, a cada ano (WHO, 2014). O uso do álcool pode levar o indivíduo ao uso de outras substâncias psicoativas, como o tabaco. A nicotina é a substância encontrada no cigarro que pode causar rapidamente dependência em seus jovens usuários e os leva-lo a manutenção do vício até a idade adulta. Para Figueiredo VC, et al. (2016) o uso precoce do fumo é um preditor que este indivíduo seja um fumante na idade adulta mesmo sem a dependência da nicotina quando jovem.

O crescente uso dessas substâncias é observado nos adolescentes, por ser mais vulneráveis a experimentação. Entre os adolescentes, o uso excessivo de álcool e o tabagismo são associados ao baixo rendimento escolar, dificuldades de relacionamentos sociais, problemas comportamentais (agressividade e impulsividade) e risco de transtornos psiquiátricos (BROOK D, et al., 2006; GALDURÓZ JC, et al., 2010; MORIHISA RS, et al., 2007).

De acordo com Organização Mundial de Saúde, o consumo mundial de álcool em 2016 foi de 6,4 litros por pessoa com 15 anos ou mais, no Brasil o consumo abusivo de álcool no mesmo ano superou a média internacional 8,9 litros por pessoa (BRASIL, 2016). O uso excessivo de álcool causa diversos males à saúde física e modifica a estrutura mental e funções cognitivas, causando deficiências de percepção e raciocínio, construção de pensamentos sem clareza gerando sensações desarmoniosas e descontrole nas ações do cotidiano (PAULO ML e GOMES FD, 2012).

Estudos realizados por instituições brasileiras na última década apontam que a idade média de experimentação do tabaco por jovens, tanto meninas como meninos é de 16 anos, ocupando o segundo 
lugar no ranking de substâncias psicoativas no país (DANTAS DRG, et al., 2017). A precocidade com que os adolescentes estão iniciando o uso do cigarro é preocupante, pois o tabagismo representa um grave problema de saúde pública e continua a ser a principal causa de morte que se pode prevenir no mundo.

Diante do exposto, o objetivo é estimar a prevalência e os fatores sociodemográficos associados ao tabagismo e ao consumo excessivo de álcool por adolescentes escolares residentes em uma cidade de pequeno porte do Brasil.

\section{MÉTODOS}

Este estudo de delineamento transversal e base escolar foi realizado na cidade de Januária, Minas Gerais, Brasil, no ano de 2017. Januária situa-se no Médio São Francisco no Norte de Minas Gerais, que tem população estimada em 2017 de 68,584 habitantes e um Índice de Desenvolvimento Humano (IDH) Médio de 0,65 (IBGE, 2010).

A população probabilística foi de 2.104 alunos de 15 a 18 anos de idade, distribuída em dez escolas da rede pública estadual de ensino. Composta por alunos matriculados do 9ำ ano do Ensino Fundamental ao $3^{\circ}$ ano do Ensino Médio das escolas públicas estaduais do município de Januária.

O processo amostral foi realizado por conglomerados, estratificando-se por escolas. Inicialmente, identificou-se o número total de escolas e turmas do 9o ano do Ensino Fundamental ao $3^{\circ}$ ano do Ensino Médio. A amostra foi distribuída de acordo com o quantitativo médio de alunos por turno, estimou-se o número de 64 turmas a serem sorteadas. Todos os alunos das turmas sorteadas foram convidados a participar do estudo.

Para o cálculo do tamanho da amostra levou-se em consideração prevalência desconhecida para o desfecho $(50 \%)$, erro tolerável de cinco pontos percentuais, nível de confiança de $95 \%$, efeito de delineamento de 2,0 , acrescentando $10 \%$ para possíveis perdas e recusas.

Definiram-se como indivíduos elegíveis para o estudo aqueles matriculados na rede estadual de ensino, que se encontravam na sala de aula no dia da coleta, com faixa etária de 15 a 18 anos e que aceitaram participar voluntariamente da pesquisa dando anuência de sua participação através da assinatura do Termo de Assentimento devidamente assinado pelos pais em duas vias, uma para o participante e outra ficando com o pesquisador.

A amostra foi avaliada em 583 alunos que frequentavam do $9^{\circ}$ ano do Ensino Fundamental ao $3^{\circ}$ ano do Ensino Médio. Desses, 241 (41,3\%) eram do sexo feminino e $342(58,7 \%)$ do sexo masculino. Com idades compreendidas de 15 e 18 anos.

Os critérios de exclusão adotados foram: (a) idade inferior a 15 anos e/ou superior a 18 anos. Considerou-se recusa o adolescente que não quis participar da pesquisa. Foi considerada perda amostral não preencher completamente o questionário.

O trabalho de campo foi realizado por acadêmicos do curso de Educação Física da Universidade Estadual de Montes Claros, Campus Januária, MG. O instrumento utilizado foi o questionário do projeto denominado "Estilo de vida dos Adolescentes Manauara" que foi adaptado para ser desenvolvido na cidade de Januária-MG.

O instrumento foi aplicado em sala de aula, na ausência do professor. O questionário auto preenchido é constituído por diferentes questões, fechadas, subdividido em Informações pessoais (sexo, idade, escolaridade materna, turno de estudo, local de residência, renda familiar mensal em salários mínimos) e informações quanto ao tabagismo e uso de álcool.

Para o consumo de álcool, os adolescentes foram questionados sobre a quantidade de doses de bebidas alcoólicas que, em geral, eles ingerem em uma única ocasião. As opções de resposta foram dicotomizadas, conforme o sexo, em menos que quatro doses para as meninas (ou menos que cinco doses para os meninos), e quatro ou mais doses para as meninas (ou cinco ou mais doses para os meninos). Foram considerados como consumo excessivo de álcool, aqueles que relataram beber quatro ou mais doses - 
meninas; ou cinco ou mais doses - meninos (SIQUEIRA L e SMITH VC, 2015). Com relação ao tabaco, a pergunta era sobre a situação atual do jovem (não fumante, ex-fumante, fumante). A verificação das variáveis sociodemográficas associadas ao tabagismo foi realizada por meio da regressão logística multinomial (desfecho politômico), sendo a categoria "não fumante" considerada como referência.

Além disso, com o objetivo de identificar a associação entre consumo de álcool e variáveis sociodemográficas, se utilizou a regressão logística binária (desfecho dicotômico). Em ambas as análises foram estimadas odds ratio e intervalo de confiança de 95\% (IC95\%). As variáveis sociodemográficas foram analisadas enquanto possíveis modificadoras de efeito, considerando neste caso um valor $p<0,10$ como indicativo de interação.

$\mathrm{Na}$ execução dos modelos ajustados das regressões, todas as variáveis foram inseridas no mesmo nível, independentemente do $p$ valor na análise bruta, permanecendo no modelo aquelas com $p$ valor $\leq 0,20$ e utilização do método backward. A estratégia utilizada para avaliar os modelos finais (regressão logística multinomial e regressão logística binária), foi a estimação de um modelo saturado, em que as interações entre todas as variáveis independentes foram incluídas, de modo que seus parâmetros de ajuste pudessem ser comparados entre si.

Ainda, um modelo nulo foi estimado (sem incorporação de variáveis independentes) para comparar os modelos finais. Entre as ferramentas de verificação de ajustes destes modelos, parâmetros como coeficiente de determinação $\mathrm{R}^{2}$, Critério de Informação de Akaike (AIC) e Critério de Informação Bayesiano $(B I C)$ foram utilizados. A análise estatística foi realizada no pacote estatístico Stata® (Statistical Software for Professionals, Texas), versão 13.0.

Os instrumentos e procedimentos propostos no estudo foram submetidos para avaliação e aprovação do Comitê de Ética da Universidade Estadual de Montes Claros- UNIMONTES, Minas Gerais, Brasil. Parecer Consubstanciado no 2.163 .521 de 08 de Julho de 2017.

\section{RESULTADOS}

No presente estudo, se verificou que mais da metade da amostra era formada por meninas $(58,6 \%)$. A maioria tinha idade de 15 a 16 anos (58,6\%), mães com até oito anos de estudo (38,9\%) e renda de até um salário mínimo (66,2\%). Do total de avaliados, 51,3\% estudava no período matutino e 93,8\% residia em área urbana. A respeito do tabagismo, 2,3\% dos escolares relataram fazer a utilização de cigarro. Ainda, $6,9 \%$ dos avaliados relatou consumo excessivo de álcool (Tabela 1). 
Tabela 1 - Distribuição da amostra em relação a fatores sóciodemográficos, tabagismo e consumo de álcool em escolares da amostra investigada.

\begin{tabular}{|c|c|c|}
\hline Variáveis & $\mathbf{n}$ & $\%$ (IC95\%) \\
\hline \multicolumn{3}{|l|}{ Sexo } \\
\hline Feminino & & $58,6(54,6-62,6)$ \\
\hline Masculino & 241 & $41,4(37,4-45,4)$ \\
\hline \multicolumn{3}{|l|}{ Idade (anos) } \\
\hline $15-16$ & 342 & $58,6(54,6-62,6)$ \\
\hline $17-18$ & 241 & $41,4(37,4-45,4)$ \\
\hline \multicolumn{3}{|l|}{ Escolaridade Materna } \\
\hline Nunca estudou & 40 & $6,9(5,0-9,2)$ \\
\hline Até oito anos completos & 227 & $38,9(35,0-42,9)$ \\
\hline Até doze anos completos & 182 & $31,2(27,5-35,1)$ \\
\hline Acima de doze anos & 134 & $23,0(19,7-26,6)$ \\
\hline \multicolumn{3}{|c|}{ Renda Mensal Familiar (salários mínimos) } \\
\hline Até um salário & 386 & $66,2(62,2-69,9)$ \\
\hline Dois ou mais salários & 197 & $33,8(30,0-37,7)$ \\
\hline \multicolumn{3}{|l|}{ Turno de estudo } \\
\hline Matutino & 299 & $51,3(47,2-55,3)$ \\
\hline Vespertino & 199 & $34,1(30,4-38,1)$ \\
\hline Noturno & 85 & $14,6(11,9-17,7)$ \\
\hline \multicolumn{3}{|l|}{ Local de residência } \\
\hline Urbana & 547 & $93,8(91,5-95,5)$ \\
\hline Rural & 36 & $6,2(4,4-8,4)$ \\
\hline \multicolumn{3}{|l|}{ Tabagismo } \\
\hline Nunca fumou & 550 & $94,3(92,1-95,9)$ \\
\hline Ex-fumante & 20 & $3,4(2,2-5,2)$ \\
\hline Fumante & 13 & $2,3(1,3-3,8)$ \\
\hline \multicolumn{3}{|l|}{ Consumo de álcool } \\
\hline Não & 543 & $93,1(90,7-94,9)$ \\
\hline Sim & 40 & $6,9(5,1-9,2)$ \\
\hline
\end{tabular}

Legenda: IC - Intervalo de Confiança. Fonte: Silva AF, et al., 2020.

$\mathrm{Na}$ análise ajustada foi encontrado que maiores chances (OR: 4,26; IC95\%: 1,31-13,82) de ser ex-fumante foram verificadas nos escolares que residiam em áreas rurais. Além disso, ser do sexo masculino (OR: 5,18; IC95\%: 1,39-19,28), estar na faixa etária de 17-18 anos (OR: 1,96; IC95\%: 1,16-3,30) e estudar no turno vespertino (OR: 7,66; IC95\%: 1,62-36,24) foi relacionado com maiores chances de ser fumante (Tabela 2). 
Tabela 2 - Distribuição da amostra de acordo com o tabagismo e análise ajustada da associação entre tabagismo com fatores sociodemográficos em escolares de 15 a 18 anos da amostra investigada.

\begin{tabular}{|c|c|c|c|c|c|}
\hline \multicolumn{6}{|c|}{ Tabagismo } \\
\hline & Não fumante & & -Fumante & & Fumante \\
\hline Variáveis $^{\mathrm{b}}$ & n (\%) & n (\%) & OR (IC95\%) ${ }^{a}$ & n (\%) & OR (IC95\%) \\
\hline \multicolumn{6}{|l|}{ Sexo } \\
\hline Feminino & $331(96,8)$ & $08(2,3)$ & 1,00 & $03(0,9)$ & 1,00 \\
\hline Masculino & $219(90,9)$ & $12(5,0)$ & $2,26(0,90-5,68)$ & $10(4,1)$ & $5,18(1,39-19,28)$ \\
\hline \multicolumn{6}{|l|}{ Idade (anos) } \\
\hline $15-16$ & $326(95,3)$ & $10(2,9)$ & 1,00 & $06(1,8)$ & 1,00 \\
\hline $17-18$ & $224(92,9)$ & $10(4,1)$ & $0,88(0,57-1,37)$ & $07(3,0)$ & $1,96(1,16-3,30)$ \\
\hline \multicolumn{6}{|l|}{ Escolaridade Materna } \\
\hline Nunca estudou & $36(90,0)$ & $02(5,0)$ & 1,00 & $02(5,0)$ & 1,00 \\
\hline Até oito anos completos & $217(95,6)$ & $06(2,6)$ & $0,56(0,10-3,09)$ & $04(1,8)$ & $0,38(0,06-2,39)$ \\
\hline $\begin{array}{l}\text { Até doze anos } \\
\text { completos }\end{array}$ & $175(96,1)$ & $05(2,7)$ & $0,62(0,10-3,62)$ & $02(1,2)$ & $0,26(0,03-2,17)$ \\
\hline Acima de doze anos & $122(91,0)$ & $07(5,2)$ & $1,24(0,23-6,77)$ & $05(3,8)$ & $0,90(0,15-5,50)$ \\
\hline \multicolumn{6}{|l|}{$\begin{array}{l}\text { Renda Mensal Familiar } \\
\text { (salários mínimos) }\end{array}$} \\
\hline Até um salário & $364(94,3)$ & $13(3,4)$ & 1,00 & $09(2,3)$ & 1,00 \\
\hline Dois ou mais salários & $186(94,4)$ & $07(3,5)$ & $0,95(0,34-2,59)$ & $04(2,1)$ & $0,69(0,19-2,52)$ \\
\hline \multicolumn{6}{|l|}{ Turno de estudo } \\
\hline Matutino & $289(96,6)$ & $08(2,7)$ & 1,00 & $02(0,7)$ & 1,00 \\
\hline Vespertino & $180(90,4)$ & $10(5,0)$ & $2,10(0,80-5,48)$ & $09(4,6)$ & $7,66(1,62-36,24)$ \\
\hline Noturno & $81(95,4)$ & $02(2,3)$ & $0,89(0,18-4,32)$ & $02(2,3)$ & $3,48(0,47-25,40)$ \\
\hline \multicolumn{6}{|l|}{ Local de residência } \\
\hline Urbana & $520(95,0)$ & $16(2,9)$ & 1,00 & $11(2,1)$ & 1,00 \\
\hline Rural & $30(83,3)$ & $\begin{array}{c}04 \\
(11,1)\end{array}$ & $\begin{array}{c}4,26(1,31- \\
13,82)\end{array}$ & $02(5,6)$ & $2,98(0,59-15,00)$ \\
\hline
\end{tabular}

Legenda: $\mathrm{OR}=$ odds ratio; $\mathrm{IC}=$ Intervalo de confiança; $\mathrm{a}=$ Análise ajustada para todas as variáveis independentes; $b=A$ categoria de referência para as análises foram os escolares que nunca fumaram. $O$ modelo final formado pelas variáveis sexo, turno de estudo e local de residência, apresentou valor de pseudo $\mathrm{R}^{2}=0,03914 ; \mathrm{AIC}=306.640$ e $\mathrm{BIC}=-3327.385$. Em comparação, o modelo saturado apresentou os seguintes indicadores de ajuste (pseudo $R^{2}=0,0701 ; A I C=319.000$ e $B I C=-3301.921$ ). $O$ modelo nulo teve estes resultados (pseudo $R^{2}=0 ; A I C=303.878 ; B I C=-3395.670$ ). Fonte: Silva $A F$, et al., 2020.

A análise bruta demonstrou que os escolares de faixa etária 17-18 anos e que estudavam no período noturno tinham, respectivamente, 101\% (OR: 2,01; IC95\%: 1,05-3,86) e 117\% mais chances de apresentarem consumo excessivo de álcool.

Também verificou se que os escolares que residiam em área rural tinham $63 \%$ de chances a menos (OR: 0,37; IC95\%: 0,25-2,78) de consumirem álcool em excesso. Na análise ajustada, os adolescentes de faixa etária 17 a 18 anos tiveram $113 \%$ mais chances de consumirem álcool de maneira excessiva (OR: 0,37; IC95\%: 0,25-2,78) (Tabela 3). 
Tabela 3 - Distribuição da amostra de acordo com consumo de álcool e associação entre consumo de álcool com fatores sociodemográficos em escolares de 15 a 18 anos da amostra investigada.

\begin{tabular}{|c|c|c|c|c|c|c|}
\hline \multicolumn{7}{|c|}{ Consumo de álcool } \\
\hline & Sim & Não & Análise Bruta & & Análise Ajustada & \\
\hline Variáveis & n (\%) & n (\%) & OR (IC95\%) $)^{a}$ & $p$ & OR (IC95\%) $)^{a}$ & $p$ \\
\hline \multicolumn{7}{|l|}{ Sexo } \\
\hline Feminino & $19(47,5)$ & $323(59,5)$ & 1,00 & 0,41 & 1,00 & 0,21 \\
\hline Masculino & $21(52,5)$ & $220(40,5)$ & $1,62(0,85-3,08)$ & & $1,51(0,78-2,90)$ & \\
\hline \multicolumn{7}{|l|}{ Idade (anos) } \\
\hline $15-16$ & $17(42,5)$ & $325(59,8)$ & 1,00 & 0,03 & 1,00 & 0,02 \\
\hline $17-18$ & $23(57,5)$ & $218(40,2)$ & $2,01(1,05-3,86)$ & & $2,13(1,10-4,11)$ & \\
\hline \multicolumn{7}{|l|}{ Escolaridade Materna } \\
\hline Nunca estudou & $04(10,0)$ & $36(6,6)$ & 1,00 & 0,87 & 1,00 & 0,98 \\
\hline Até oito anos completos & $14(35,0)$ & $213(39,2)$ & $0,59(0,18-1,89)$ & & $0,51(0,15-1,69)$ & \\
\hline Até doze anos completos & $11(27,5)$ & $171(31,5)$ & $0,58(0,17-1,92)$ & & $0,49(0,13-1,72)$ & \\
\hline Acima de doze anos & $11(27,5)$ & $123(22,7)$ & $0,80(0,24-2,68)$ & & $0,67(0,19-2,43)$ & \\
\hline \multicolumn{7}{|l|}{$\begin{array}{l}\text { Renda Mensal Familiar } \\
\text { (salários mínimos) }\end{array}$} \\
\hline Até um salário & $23(57,5)$ & $363(66,8)$ & 1,00 & 0,23 & 1,00 & 0,14 \\
\hline Dois ou mais salários & $17(42,5)$ & $180(33,2)$ & $1,49(0,77-2,86)$ & & $1,63(0,84-3,16)$ & \\
\hline \multicolumn{7}{|l|}{ Turno de estudo } \\
\hline Matutino & $21(52,5)$ & $278(51,2)$ & 1,00 & 0,20 & 1,00 & 0,75 \\
\hline Vespertino & $07(17,5)$ & $192(35,3)$ & $0,48(0,20-1,15)$ & & $0,43(0,17-1,04)$ & \\
\hline Noturno & $12(30,0)$ & $73(13,5)$ & $2,17(1,02-4,62)$ & & $1,47(0,62-3,47)$ & \\
\hline \multicolumn{7}{|l|}{ Local de residência } \\
\hline Urbana & $39(97,5)$ & $508(93,5)$ & 1,00 & 0,33 & 1,00 & 0,26 \\
\hline Rural & $01(2,5)$ & $35(6,5)$ & $0,37(0,25-2,78)$ & & $0,31(0,21-2,38)$ & \\
\hline
\end{tabular}

Legenda: $\mathrm{OR}=$ odds ratio; $\mathrm{IC}=$ Intervalo de confiança; $\mathrm{a}=$ Análise ajustada para todas as variáveis independentes; O modelo final formado pelas variáveis idade e renda mensal familiar, apresentou valor de pseudo $\mathrm{R}^{2}=0,0227 ; \mathrm{AIC}=290.929$ e BIC $=-3408.619$. Em comparação, o modelo saturado apresentou os seguintes indicadores de ajuste (pseudo $R^{2}=0,0343 ; A I C=295.536$ e $B I C=-3386.539$ ). $O$ modelo nulo teve estes resultados (pseudo $\mathrm{R}^{2}=0 ; \mathrm{AIC}=298.551 ; \mathrm{BIC}=-314.767$ ). Fonte: Silva $A F$, et al., 2020.

\section{DISCUSSÃO}

A adolescência é uma fase da vida humana que está entre a infância e a vida adulta, com forte desenvolvimento emocional, físico, sexual, e social. Inicia-se com as mudanças físicas da puberdade culminando na consolidação do crescimento corporal. A adolescência é a fase em que o indivíduo começa sua busca em alcançar seus objetivos rumo às expectativas da sociedade onde está inserido (TANNER JM, 1962).

O presente estudo estimou a prevalência e os fatores sociodemográficos associados ao tabagismo e ao consumo de álcool em adolescentes escolares da cidade de Januária, MG. A prevalência estimada para tabagismo e consumo de álcool foi de 2,3\% e 6,9\% respectivamente. Além disso, maiores chances para a condição "ex-fumante" foi verificada nos escolares residentes em áreas rurais.

Os escolares do sexo masculino e de faixa etária elevada (17-18 anos) tiveram maiores chances de serem fumantes quando comparados aos escolares do sexo feminino e àqueles de menor faixa etária (1516 anos). Em relação ao consumo de álcool, escolares de faixa etária elevada (17-18 anos) apresentaram maiores chances para esta condição em comparação aos escolares de menor faixa etária (15-16 anos).

A prevalência estimada para tabagismo no presente estudo foi de 2,9\%. Este resultado é semelhante ao identificado no estudo com amostra representativa de escolares brasileiros, em que a prevalência estimada 
para tabagismo entre adolescentes (12 a 17 anos de idade) foi de 2,5\% (FIGUEIREDO VC, et al., 2016). A reduzida prevalência de escolares fumantes na presente pesquisa poderia estar relacionada as constantes campanhas e ações direcionadas ao controle do tabagismo no Brasil (LEVY D, et al., 2012).

Embora os resultados do presente estudo tenham revelado baixa prevalência de escolares positivos para tabagismo, estes achados devem ser observados com cautela, uma vez que o hábito de fumar em geral tem seu início durante a adolescência, e a probabilidade de jovens fumantes permanecerem com este hábito quando adultos é elevada (FIGUEIREDO VC, et al., 2016).

A prevalência de consumo de álcool entre escolares neste estudo $(6,9 \%)$ foi menor que a identificada em pesquisa realizada com escolares ( 14 a 17 anos de idade) de diversas regiões brasileiras (PINSKY I, et al., 2010), que identificou prevalência de $48,4 \%$ para o consumo de álcool.

Na pesquisa realizada com escolares de 13 a 19 anos de idade na cidade de Olinda/PE, a prevalência estimada para consumo de álcool foi de 23,1\% (RAPOSO JCS, et al., 2017). Embora a venda de bebidas alcoólicas para indivíduos com idade inferior aos 18 anos seja proibida no Brasil, muitos adolescentes brasileiros consomem álcool (BARBOSA FILHO VC, et al., 2012).

Contudo, hipotetiza-se que a omissão dos escolares em respeito de experiências passadas relacionando o consumo abusivo de drogas lícitas não permitidas para adolescentes $(<18$ anos), possa ter implicado para a reduzida prevalência de consumo de álcool no presente estudo.

Esses achados são preocupantes pois o crescente consumo de álcool por adolescentes pode levar a várias complicações cerebrais, podendo desencadear problemas psicológicos, como a ansiedade, estresse, depressão, insônia, dentre outros. Também pode acarretar várias doenças como a gastrite, úlceras, hepatites, anemia, Hipertensão arterial, cirrose, pancreatite e acidentes vasculares, podendo levar até a morte (SANTOS VS, 2020).

Os adolescentes residentes em área rural apresentaram maiores chances de serem ex-fumantes em comparação àqueles que moravam em área urbana. As áreas rurais são afetadas pela ausência/escassez de opções de lazer e cultura, prática de esportes e ambientes de convívio públicos, o que pode implicar negativamente na vida dos adolescentes e contribuir para adoção de hábitos de vida não saudáveis, como o tabagismo (BRASIL, 2010).

A possível justificativa para os achados referentes ao local de moradia (área rural) e adolescentes exfumantes poderia estar relacionado ao aumento das áreas de abrangência dos meios de comunicação (importantes disseminadores de políticas de enfrentamento de hábitos de vida inadequados) não somente em regiões periféricas dos grandes centros urbanos, mas também nas zonas rurais, o que pode ter contribuído para que os adolescentes antes positivos para tabagismo cessassem o seu consumo.

Os achados do presente estudo identificaram que os meninos tiveram $418 \%$ mais chances de serem fumantes em comparação as meninas. Estes resultados divergem de outros estudos que não identificaram diferenças para tabagismo entre os sexos (BONILHA AG, et al., 2014; FIGUEIREDO VC, et al., 2016). Durante a adolescência, os indivíduos são mais susceptíveis as influências sociais e ambientais (VITORIA PD, et al., 2013; FIGUEIREDO VC, et al., 2016).

Vários são os fatores que levam a experimentar o cigarro ou outros derivados do tabaco. A curiosidade pelo produto, imitação do comportamento do adulto, necessidade de auto-afirmação e encorajamento proporcionado pela aceitação em grupos de interesse com padrões pré-estabelecidos, pais, professores, ídolos e amigos podem levar os adolescentes a iniciar o uso de cigarros (SESP, 2007).

Os escolares de faixa etária elevada (17-18 anos) tiveram maiores chances de serem fumantes em comparação aos de menor faixa etária (15-16 anos). Estes achados estão de acordo com a literatura, que identificou maior adoção de comportamentos de risco à saúde, como ser fumante, entre adolescentes de maior faixa etária em comparação aos de menor faixa etária fumante, entre adolescentes de maior faixa etária em comparação aos de menor faixa etária (BRITO ALS, et al., 2015; FIGUEIREDO VC, et al., 2016). Possível justificativa para as maiores chances de ser fumante entre escolares de maior faixa etária em 
comparação aos de menor faixa etária poderia estar relacionado a aquisição de maior independência, autonomia social e econômica concomitante ao acréscimo dos anos de vida, o que favorece o acesso a ambientes cuja adoção de comportamentos de risco são menos recriminados (EISENSTEIN E, 2005).

Surpreendentemente, apenas o turno de estudo vespertino esteve associado a maiores chances de os adolescentes serem fumantes. Ainda que a relação entre turno de estudo e tabagismo em adolescentes brasileiros tenha sido pouco investigada a associação identificada no presente estudo é incomum (BARBOSA FILHO VC, et al., 2012). Escolares do turno de estudo noturno, em geral, são mais velhos, tem menor nível econômico e tendem a adotar hábitos de risco próximos aos dos adultos, como o consumo de cigarro ( BARBOSA FILHO VC, et al., 2012; CASTRO JAC, et al., 2016). Desta forma, maior número de inquéritos cujo objetivo seja investigar a relação entre turno de estudo e tabagismo em adolescentes se fazem necessários para confirmar o direcionamento dos achados do presente estudo.

Verificou-se neste estudo que os escolares de maior faixa etária tiveram maiores chances para o consumo de álcool em comparação aos de menor faixa etária. O estudo realizado com participação de 661 escolares (14 a 17 anos de idade) de distintas regiões do Brasil também identificou que adolescentes de maior faixa etária eram mais propícios ao consumo de álcool quando comparados aos de menor faixa etária (PINSKY I, et al., 2010).

A Possível justificativa em relação às maiores chances de consumo de álcool para os adolescentes de maior faixa etária estaria relacionada ao fato de que concomitante ao aumento dos anos de vida dos adolescentes, maior o número de eventos propícios para o consumo abusivo de álcool, como reuniões de famílias, amigos, festas públicas ou particulares, "open bar", soma-se a isso as campanhas publicitárias com propagandas atrativas, criativas com o objetivo de conquistar cada vez mais o público jovem, o que poderia implicar diretamente no consumo abusivo de bebidas alcoólicas (PINSKY e EL JUNDI, 2008).

Como limitação deste estudo, o delineamento transversal, que impede o estabelecimento de causalidade temporalidade das associações testadas. A investigação das informações por meio de questionário autoadministrado, o que possibilita viés de resposta por parte dos avaliados também é considerada limitação da presente pesquisa. Contudo, este estudo apresenta contribuição importante para a área da saúde, pois identificou grupos suscetíveis para tabagismo e consumo de álcool.

A escola possui papel importante no comportamento do escolar, pois também estimula a propagação de hábitos e atitudes saudáveis. Desta forma, intervenções em ambiente escolar com o objetivo de orientar e conscientizar os escolares a respeito dos efeitos nocivos a saúde atribuídos a adoção de comportamento de risco à saúde devem ser reforçadas.

\section{CONCLUSÃO}

Os resultados encontrados neste estudo demonstram que a prevalência estimada para tabagismo foi de $2,3 \%$, enquanto para o consumo de álcool, a prevalência estimada foi de $6,9 \%$. Embora esses resultados sejam menores do que a média nacional, são preocupantes, pois pode trazer consequências negativas a curto e longo prazo para os adolescentes. Essas informações são relevantes e evidenciam a necessidade de desenvolvimento e implementação de políticas de fomento à pesquisa nesta região do norte de Minas Gerais, com o propósito de desenvolver programas e estratégias de ação para promover uma população escolar mais saudável, distante de vícios como tabagismo e alcoolismo, premiando comportamentos ativos voltados para a saúde mental e física.

\section{REFERÊNCIAS}

1. BARBOSA FILHO VC, et al. Prevalence of alcohol and tobacco use among Brazilian adolescents: a systematic review. Revista de Saúde Pública, 2012 v. 46, n. 5, p. 901-917.

2. BONILHA AG, et al. Correlates of experimentation with smoking and current cigarette consumption among adolescents. Jornal Brasileiro de Pneumologia, 2014. v. 40, n. 6, p. 634-642. 
3. BRASIL. Ministério da saúde. Diretrizes nacionais para a atenção integral à saúde de adolescentes e jovens na promoção, proteção e recuperação da saúde: Ministério da Saúde Brasília 2010. [acesso 2018 junho 21]. Dísponivel

em: http://bvsms.saude.gov.br/bvs/publicacoes/diretrizes_nacionais_atencao_saude_adolescentes_jovens_promocao_s aude.pdf

4. BRASIL. Ministério da Saúde. Secretaria de Vigilância em Saúde. Vigitel Brasil: vigilância de fatores de risco e proteção para doenças crônicas por inquérito telefônico. Brasília: Ministério da Saúde, 2016.

5. BRITO ALS, et al. Prevalência e fatores associados à simultaneidade de comportamentos de risco à saúde em adolescentes. Revista Paulista de Pediatria, 2015.v. 33, n. 4, p. 423-430.

6. BROOK DW, et al. Cigarette smoking in the adolescent children of drug-abusing fathers. Pediatrics 2006; $117: 1339$ 47.

7. CARDOSO FM, et al. Fatores associados à prática do binge drinking entre estudantes da área da saúde. Rev. CEFAC 2015. vol.17 no.2 São Paulo Mar./Apr.

8. CASTRO JAC, et al. Prevalência de obesidade abdominal em adolescentes: associação entre fatores sociodemográficos e estilo de vida. Revista Paulista de Pediatría, 2016 v. 34, n. 3, p. 343-351.

9. CEBRID. Universidade Federal de São Paulo. Centro Brasileiro de Informações Sobre Drogas Psicotrópicas. Departamento de Psicobiologia - UNIFESP/EPM. São Paulo, 2018.

10. DANTAS DRG, et al. Prevalência e Risco de Tabagismo entre Estudantes do Ensino Médio em cidade do Nordeste do Brasil. Port J Public Health, 2017 vol.35 no.1 Basel set.

11. EISENSTEIN E. Adolescência: definições, conceitos e critérios. Adolescência e Saúde, 2005 v. 2, n. 2 , p. 6-7.

12. FIGUEIREDO VC, et al. ERICA: prevalência de tabagismo em adolescentes brasileiros. Revista de Saúde Pública, 2016. v. 50, n. suppl. 1, p. 12.

13. GALDURÓZ JC, et al. Fatores associados ao uso pesado de álcool entre estudantes das capitais brasileiras. Rev Saúde Pública 2010; 44:267-73.

14. INSTITUTO BRASILEIRO DE GEOGRAFIA E ESTATÍSTICA (IBGE). População e Economia do Município de Januária. Minas Gerais: IBGE; 2010. [acessado 2017 Julho 15]. Disponível em: https://cidades.ibge.gov.br/brasil/mg/januaria/panorama

15. LEVY D, et al. The Brazil SimSmoke policy simulation model: the effect of strong tobacco control policies on smoking prevalence and smoking-attributable deaths in a middle income nation. PLoS medicine, 2012 v. 9, n. 11, p. e1001336.

16. MORIHISA RS, et al. Labeling disorder - the relationship between conduct problems and drug use in adolescents. Rev Bras Psiquiatr 2007; 29:308-14.

17. OPAS/OMS-BRASIL. Setor de Embaixadas Norte. Lote 19. Brasília-DF: Brasil. Julho, 2019.

18. PAULA ML, GOMES FD. ÁLCOOL, Jurisdição, controle social e violência. Rev. Ciências Juridicas e Sociais. CROCE, Delton. Manual de Medicina Legal. 8aㅡ ed. 2012. São Paulo: Saraiva. 119p.

19. PINSKY I, et al. O impacto da publicidade de bebidas alcoólicas sobre o consumo entre jovens: revisão da literatura internacional. Revista Brasileira de Psiquiatria. v.30, n.4, p. 362 -374, 2008.

20. PINSKY I, et al. Patterns of alcohol use among Brazilian adolescents. Rev. Brasileira de Psiquiatria, 2010 v. 32 , n. 3, p. 242-249.

21. RAPOSO JCS, et al. Uso de drogas ilícitas e binge drinking entre estudantes adolescentes. Revista de Saúde Pública, 2017. 2017 v. 51, p. 1-7.

22. SANTOS VS. "Riscos do consumo de bebidas alcoólicas"; Brasil Escola. Disponível em: https://brasilescola.uol.com.br/saude-na-escola/riscos-consumo-bebidas-alcoolicas.htm. Acesso em 19 de março de 2020.

23. SECRETARIA DE ESTADO DE SEGURANÇA PÚBLICA. Drogas! Conhecer para prevenir. Curso/treinamento de Extensão Universitária em Prevenção às Drogas e Violência. Amazonas, 2007.

24. SIQUEIRA L, SMITH VC. Committee On Substance Abuse. Binge Drinking. Pediatrics. 2015 ;136(3):e718-26.

25. TANNER JM. Growth at Adolescence. 2 ed. Oxford: Blackwell, 1962.

26. VITORIA PD, et al. Determinantes do comportamento tabágico e implicações para a prevenção do tabagismo. Revista de Psicologia da Criança e do Adolescente, 2013 v. 4, n. 1, p. 191-206.

27. WORLD HEALTH ORGANIZATION. Global status report on alcohol and health. Geneva: World Health Organization; 2014. 